\title{
Влияние примесных компонентов на осаждение солей кальция в процессе очистки фосфорной кислоты на анионите методом удерживания кислоты
}

\author{
(C) 2021 Казначеев M.А. ${ }^{1}$, Тихонов Н.А. ${ }^{1}$, Хамизов P.X. ${ }^{2}$ \\ ${ }^{1}$ Московский государственный университет им. М.В. Ломоносова, Москва \\ ${ }^{2}$ Институт геохимии и аналитической химии им. В. И. Вернадского РАН, Москва
}

Поступила в редакцию 15.06.2021 г.

DOI: $10.17308 /$ sorpchrom.2021.21/3639

\begin{abstract}
Метод удерживания кислоты в сорбенте (Acid Reatardation) является эффективным способом для разделения смесей кислот и солей, основанным на различии в размерах молекул кислот и гидратированных ионных пар солевых компонентов, образующихся в сильно концентрированных растворах. Метод заключается в том, что раствор смеси компонентов с общим анионом пропускается через колонку с гелевым анионитом, находящимся в той же анионной форме, что позволяет исключить ионный обмен. На первом этапе процесса кислота задерживается внутри нанопор сорбента, в то время как раствор солей проходит через слой. Этот процесс продолжается до тех пор, пока не прекратится удерживание кислоты, и она не появится на выходе из колонки. Далее осуществляется второй этап - вытеснение кислоты промывкой слоя в колонке водой (классический метод) или специальным промывочным раствором (модифицированный метод). При этом на выходе получается раствор чистой кислоты с высокой концентрацией. В настоящей работе анализируются результаты эксперимента по очистке промышленной экстракционной фосфорной кислоты (ЭФК), содержащей большое количество примесей, модифицированным методом, в котором использование раствора разбавленной фосфорной кислоты в качестве вытесняющего раствора позволяет предотвратить осаждение солей кальция. Для исследования влияния промывочного раствора на процесс осаждения в работе сформулирована математическая модель химического равновесия в системе из ограниченного числа компонентов ЭФК, которая позволяет рассчитать молекулярный и ионный состав смеси в зависимости от концентраций входных веществ, в том числе определить количество вещества в осадке. Показано, что в случае, когда в исходном растворе находятся только соли кальция и фосфорная кислота, концентрации промывочного раствора 0.1 моль/дм по фосфорной кислоте достаточно, чтобы предотвратить осаждение, и этот эффект обусловлен образованием более растворимых кислых солей. Если в растворе также присутствуют соли других металлов, в частности алюминия и железа, то как предсказывает модель, механизм образования кислых солей не объясняет отсутствия осадкообразования. Для этого потребовалась бы концентрация промывочного раствора не менее 0.13 моль/дм³. Результаты моделирования позволяют сделать вывод, что в системе имеются другие механизмы, препятствующие осаждению. Одним из таких механизмов может являться эффект изотермического пересыщения в слое ионита.
\end{abstract}

Ключевые слова: метод удерживания кислоты, разделение солей и кислот, химическое равновесие, математическое моделирование, эффект изотермического пересыщения.

\section{Введение}

В многих промышленных технологических процессах, включая процессы гидрометаллургии, производства быстрорастворимых удобрений, а также при переработке промышленных сточных вод возникает необходимость проводить разделение концентрированных смесей солей и кислот. Существует несколько перспективных способов реализации данной процедуры, одним из которых является метод удерживания кислоты [1-4]. Он заключается в том, что смесь солей и кислот с общим анионом пропускается через 
колонку с гелевым анионитом, находящимся в той же анионной форме, что исключает ионный обмен. На первом этапе процесса кислота, которая в концентрированных растворах находится преимущественно в форме сильно связанных ионных пар или молекул, проникает наноразмерные поры и задерживается в них, в то время как солевые компоненты, гидратированные ионы которых имеют большие размеры, проходят через слой [5-8]. Этот процесс продолжается до тех пор, пока не прекратится удерживание кислоты и она не появится на выходе колонки. Далее осуществляется второй этап - промывка слоя в колонке водой. При этом на выходе получается раствор с высокой концентрацией кислоты.

Основным преимуществом данного метода являются его простота и дешевизна, а в качестве единственного реагента, затрачиваемого в процессе, выступает вода. Но данный метод имеет существенные ограничения. На первом этапе процесса, за счет задержки кислоты внутри микропор сорбента, ее концентрация в проходящем растворе существенно падает. При этом значительная часть солей, концентрационные фронты которых при опережающем движении попадают в нейтральную среду, могут перейти в плохо растворимые формы. Возможно выпадение осадка и кольматация сорбционного слоя. Поэтому метод удерживания кислоты в его классическом варианте
(Acid Retardation) [1-3] применим только к модельным растворам и некоторым реальным объектам, в которых не содержатся компоненты, которые могут образовать труднорастворимые осадки, такие как сульфат и средний фосфат кальция, фосфаты и гидратированные оксиды других компонентов.

В работах $[5,8]$ был предложен «модифицированный способ удерживания кислоты» (Modified Acid Retardation) и продемонстрирован способ избежать осадкообразования, в частности, образования осадка солей кальция при очистке промышленной фосфорной кислоты. Для этого в каждом текущем цикле процесса поглощения-вытеснения кислоты в качестве вытесняющего агента вместо дистиллированной воды было предложено использовать разбавленную чистую кислоту, которая получается в хвостовых фракциях элюирования на стадии вытеснения предыдущего цикла. Например, в лабораторных и пилотных экспериментах по очистке ЭФК концентрация фосфорной кислоты в вытесняющем растворе, достаточная для препятствия процессу осаждения, оказалась не более 0.1 моль/дм ${ }^{3}$ в то время, как концентрация кислоты в исходной смеси подлежащей разделению и ее концентрация в «очищенной» части составляла 2.55 моль/дм³

На рисунке 1 представлены экспериментальные результаты из работы [8].

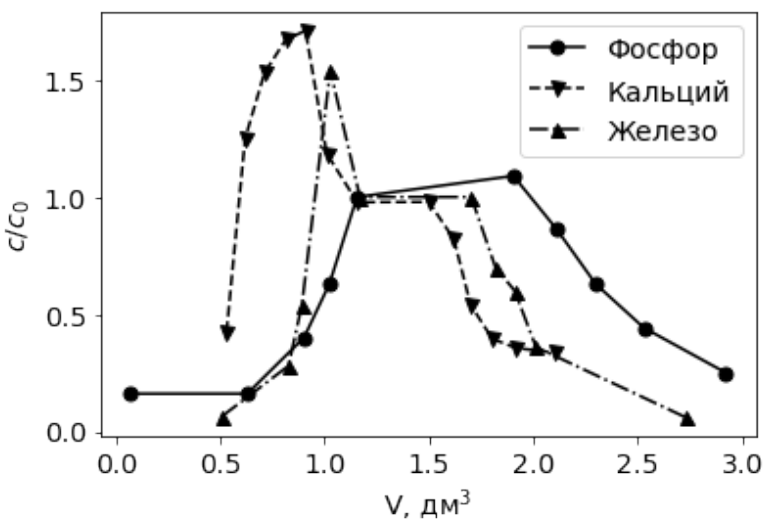

Рис. 1. Концентрации на выходе из колонки в эксперименте [8], нормированные на входные концентрации.

Fig. 1. Concentrations at the outlet of the column in the experiment [8] normalised to the inlet concentrations. 
Изображены выходные кривые концентрации солей и кислоты, нормированные на входные концентрации. На первом этапе из колонки выходит раствор несущий основное количество солей, далее осуществляется стадия вытеснения (далее, для простоты будем использовать термин «промывка»). При промывке выходит основная часть кислоты. Цикл повторяется.

Отсутствие осадка можно объяснить действием двух факторов: переходом солей в более растворимую форму благодаря небольшому количеству фосфорной кислоты в промывочном растворе и благодаря эффекту изотермического пересыщения $[9,10]$. В настоящей работе будет показано, что при наличии в растворе солей железа (и алюминия) отсутствие осадка нельзя объяснить только действием первого фактора. Моделирование показывает, что для объяснения отсутствия осадка необходим учет эффекта изотермического пересыщения.

\section{Теоретическая часть}

Для того, чтобы определить, при каких концентрациях веществ соли кальция выпадают в осадок, необходимо определить молекулярный и ионный состав смеси при химическом равновесии. Для этого потребуются соответствующие уравнения химического равновесия (1-9), уравнения материального баланса (10-12) и уравнение электронейтральности (сохранения заряда) (13). Для определения количества осажденного вещества используется произведение растворимости. В модели будем рассматривать следующие компоненты: фосфорную кислоту, а также соли: средние и кислые фосфаты кальция и железа.

Тогда система уравнений будет иметь следующий вид.

$$
\begin{aligned}
K_{a 1} & =\frac{\left[\mathrm{H}_{2} \mathrm{PO}_{4}^{-}\right]\left[\mathrm{H}^{+}\right]}{\left[\mathrm{H}_{3} \mathrm{PO}_{4}\right]} \\
K_{a 2} & =\frac{\left[\mathrm{HPO}_{4}^{2-}\right]\left[\mathrm{H}^{+}\right]}{\left[\mathrm{H}_{2} \mathrm{PO}_{4}^{-}\right]} \\
K_{a 3} & =\frac{\left[\mathrm{PO}_{4}^{3-}\right]\left[\mathrm{H}^{+}\right]}{\left[\mathrm{HPO}_{4}^{2-}\right]}
\end{aligned}
$$

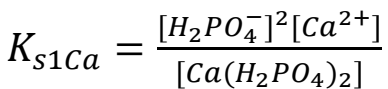

$$
\begin{aligned}
& K_{S 2 \mathrm{Ca}}=\frac{\left[\mathrm{HPO}_{4}^{2-}\right]\left[\mathrm{Ca}^{2+}\right]}{\left[\mathrm{CaHPO}_{4}\right]} \\
& K_{\text {S3Ca }}=\frac{\left[\mathrm{PO}_{4}^{3-}\right]^{2}\left[\mathrm{Ca}^{2+}\right]^{3}}{\left[\mathrm{Ca}_{3}\left(\mathrm{PO}_{4}\right)_{2}\right]} \\
& K_{\text {S1Fe }}=\frac{\left[\mathrm{H}_{2} \mathrm{PO}_{4}^{-}\right]^{3}\left[\mathrm{Fe}^{3+}\right]}{\left[\mathrm{Fe}\left(\mathrm{H}_{2} \mathrm{PO}_{4}\right)_{3}\right]} \\
& K_{S 2 F e}=\frac{\left[\mathrm{HPO}_{4}^{2-}\right]^{3}\left[\mathrm{Fe}^{3+}\right]^{2}}{\left[\mathrm{Fe}_{2}\left(\mathrm{HPO}_{4}\right)_{3}\right]} \\
& K_{\mathrm{S} 3 \mathrm{Fe}}=\frac{\left[\mathrm{PO}_{4}^{3-}\right]\left[\mathrm{Fe}^{3+}\right]}{\left[\mathrm{FePO}_{4}\right]} \\
& {\left[\mathrm{H}_{3} \mathrm{PO}_{4}\right]+\left[\mathrm{H}_{2} \mathrm{PO}_{4}^{-}\right]+\left[\mathrm{HPO}_{4}^{2-}\right]+}
\end{aligned}
$$

Представленная система нелинейных уравнений является замкнутой, дополнительными ограничениями являются положительные значения концентраций и условия растворимости компонентов (1417):

$$
\begin{array}{r}
K_{s p 1 C a} \geq\left[\mathrm{H}_{2} \mathrm{PO}_{4}^{-}\right]^{2}\left[\mathrm{Ca}^{2+}\right](14) \\
K_{s p 2 \mathrm{Ca}} \geq\left[\mathrm{HPO}_{4}^{2-}\right]\left[\mathrm{Ca}^{2+}\right](15) \\
K_{\text {sp } 3 \mathrm{Ca}} \geq\left[\mathrm{PO}_{4}^{3-}\right]^{2}\left[\mathrm{Ca}^{2+}\right]^{3}(16) \\
K_{\text {sp } 3 \mathrm{Fe}} \geq\left[\mathrm{PO}_{4}^{3-}\right]\left[\mathrm{Fe}^{3+}\right](17)
\end{array}
$$

Кислые фосфаты железа предполагаются существенно более растворимыми, чем соответствующие фосфаты кальция [11].

В таблице 1 представлены концентрационные константы диссоциации, при которых проводились расчеты. Константы для кальция рассчитаны на основе известных данных о произведениях растворимости и растворимости солей кальция из [7]. Некоторые из констант диссоциации для железа рассчитаны на основе известных значений для промежуточных реакций в предположении, что известные константы необходимо возвести в степень, соответствующую стехиометрическим коэффициентам искомой реакции. 
Таблица 1. Константы диссоциации и произведения растворимости.

Table 1. Dissociation constants and solubility products.

\begin{tabular}{|c|c|c|}
\hline Константа & Величина & Источник \\
\hline $\mathrm{K}_{\mathrm{a} 1}$ & 0.007 & {$[12]$} \\
\hline $\mathrm{K}_{\mathrm{a} 2}$ & $6.24 \cdot 10^{-8}$ & {$[12]$} \\
\hline $\mathrm{K}_{\mathrm{a} 3}$ & $4.5 \cdot 10^{-13}$ & {$[12]$} \\
\hline $\mathrm{K}_{\mathrm{s} 1 \mathrm{Ca}}$ & 0.004 & {$[13]$} \\
\hline $\mathrm{K}_{\mathrm{s} 2 \mathrm{Ca}}$ & $1.8 \cdot 10^{-4}$ & {$[13]$} \\
\hline $\mathrm{K}_{\mathrm{s} 3 \mathrm{Ca}}$ & $5.17 \cdot 10^{-24}$ & {$[13]$} \\
\hline $\mathrm{K}_{\mathrm{s} 1 \mathrm{Fe}}$ & $6.6 \cdot 10^{-9}$ & {$[14]$} \\
\hline $\mathrm{K}_{\mathrm{s} 2 \mathrm{Fe}}$ & $2.4 \cdot 10^{-34}$ & {$[15]$} \\
\hline $\mathrm{K}_{\mathrm{s} 3 \mathrm{Fe}}$ & $1.26 \cdot 10^{-22}$ & {$[13]$} \\
\hline $\mathrm{K}_{\mathrm{sp} 1 \mathrm{Ca}}$ & 0.001 & {$[13]$} \\
\hline $\mathrm{K}_{\mathrm{sp} 2 \mathrm{Ca}}$ & $2.7 \cdot 10^{-7}$ & {$[13]$} \\
\hline $\mathrm{K}_{\mathrm{sp} 3 \mathrm{Ca}}$ & $2.0 \cdot 10^{-29}$ & {$[13]$} \\
\hline $\mathrm{K}_{\mathrm{sp} 1 \mathrm{Fe}}$ & $1.3 \cdot 10^{-22}$ & \\
\hline
\end{tabular}

Концентрации некоторых компонентов в исходном растворе, использованном в эксперименте [8] представлены в таблице 2. В этом растворе кроме железа присутствует заметное количество солей алюминия. В рассматриваемых условиях системы: $\mathrm{FePO}_{4}-\mathrm{H}_{3} \mathrm{PO}_{4}-\mathrm{H}_{2} \mathrm{O}$ [11] и $\mathrm{AlPO}_{4}-\mathrm{H}_{3} \mathrm{PO}_{4}-\mathrm{H}_{2} \mathrm{O}$ [16] ведут себя похожим образом (имеют одинаковую валентность и близкие равновесные параметры и растворимость). Для того, чтобы избежать громоздких расчетов и упростить описание, мы их условно объединяем в один компонент - исходная концентрация которого условно обозначена выше как $\mathrm{C}_{\mathrm{Fe}, 0}$ и равна сумме концентраций железа и алюминия. Такой точности нам вполне хватит для доказательства основных положений данной работы.

Из эксперимента известны значения $\mathrm{C}_{\mathrm{P}, 0}, \mathrm{C}_{\mathrm{Ca}, 0}, \mathrm{C}_{\mathrm{Fe}, 0}$ в исходном растворе, которые являются суммарными концентрациями данного химического элемента, находящегося в составе какой-либо молекулы или иона.

Расчеты проводились для первого этапа рассматриваемого процесса. На этом этапе в растворе значения $\mathrm{C}_{\mathrm{Ca}}$ и $\mathrm{C}_{\mathrm{Fe}}$ на выходе меняются (рис. 1), благодаря удерживанию при молекулярной сорбции в нанопорах сорбента. Задачей моделирования было проанализировать, при каком значении $\mathrm{C}_{\mathrm{P}, 0}$ будет происходить образование осадка в наихудшем случае, в моменты, когда $\mathrm{C}_{\mathrm{Ca}}$ и $\mathrm{C}_{\mathrm{Fe}}$ максимальные. Для этого в процессе расчетов последовательно уменьшалась величина $\mathrm{C}_{\mathrm{P}, 0}$, остальные параметры оставались неизменными. При каждом значении $\mathrm{C}_{P, 0}$ определялся ионный и молекулярный состав раствора.

Численный алгоритм заключается в следующем. Все соединения были выражены через концентрации $\left[\mathrm{H}_{3} \mathrm{PO}_{4}\right]$, $\left[\mathrm{H}^{+}\right],\left[\mathrm{Ca}^{2+}\right],\left[\mathrm{Fe}^{3+}\right]$, что позволяет получить систему меньшей размерности и упрощает проведение численных расчетов. Далее система уже из 4 нелинейных уравнений решалась методом Ньютона [17]. После чего проверялись условия на растворимость соединений, в случае их неудовлетворения часть вещества переводилась в осадок. Процесс повторялся

Таблица 2. Суммарные концентрации элементов в исходном растворе.

Table 2. Total concentrations of elements in the initial solution.

\begin{tabular}{|c|c|}
\hline & Величина, моль/дм $^{3}$ \\
\hline $\mathrm{C}_{\mathrm{p}, 0}$ & 2.55 \\
\hline $\mathrm{C}_{\mathrm{Ca}, 0}$ & 0.032 \\
\hline $\mathrm{C}_{(\mathrm{Fe}+\mathrm{Al}), 0}=\mathrm{C}_{\mathrm{Fe}, 0}$ & 0.194 \\
\hline
\end{tabular}



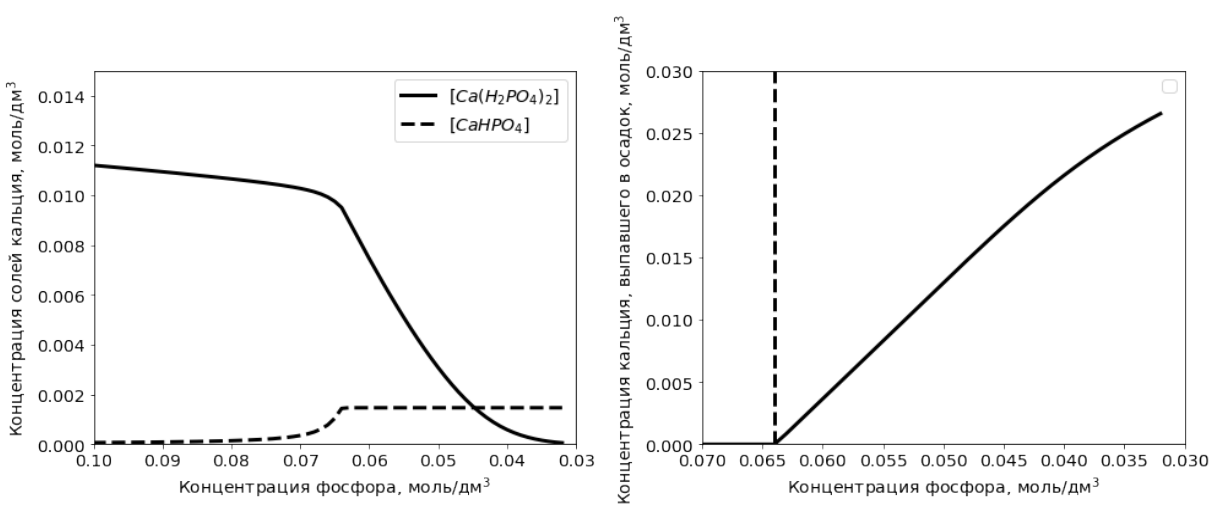

Рис. 2. Результаты моделирования в отсутствии солей железа.

Fig. 2. Simulation results in the absence of iron salts.

итеративно, пока не будут удовлетворены все уравнения в модели с некоторой заданной точностью.

\section{Обсуждение результатов}

На рисунке 2 представлены результаты для смеси фосфорной кислоты и солей кальция без других компонентов.

Можно видеть, как при уменьшении $\mathrm{C}_{\mathrm{P}, 0}$ в растворе уменьшается $\left[\mathrm{Ca}\left(\mathrm{H}_{2} \mathrm{PO}_{4}\right)_{2}\right]$ (левый график рисунка 2), увеличивается $\left[\mathrm{CaHPO}_{4}\right]$, при дальнейшем уменьшении количества фосфора $\left[\mathrm{CaHPO}_{4}\right]$ также начинает уменьшаться, увеличивается $\left[\mathrm{Ca}_{3}\left(\mathrm{PO}_{4}\right)_{2}\right]$ (последний не показан на рис. 2). При определенной концентрации $\mathrm{C}_{\mathrm{P}, 0}$ начинается процесс осаждения (вертикальная черта на правом графике рисунка 2). Видно, что при заданном количестве кальция осаждение начинается при очень низких значениях $\mathrm{C}_{\mathrm{P}, 0}\left(0.064\right.$ моль/дм $\left.{ }^{3}\right)$. Из этого можно сделать вывод, что, если бы в смеси через колонку с сорбентом пропускались бы только фосфорная кислота и только соли кальция, то концентрации 0.1 моль/дм ${ }^{3}$ фосфорной кислоты в промывочном растворе было бы достаточно, чтобы образовать более растворимые кислые соли и при этом отсутствовал бы осадок. Иными словами, отсутствие осаждения объяснялось бы только образованием кислых солей, и привлечение эффекта изотермического пересыщения не требовалось бы.

На рисунке 3 представлены результаты расчета химического равновесия с заданной суммарной концентрацией железа и алюминия.

Как и в случае с кальцием можно наблюдать изменения в молекулярном составе солей железа при уменьшении количества фосфора (левый график рисунка 3). Сначала $\mathrm{Fe}\left(\mathrm{H}_{2} \mathrm{PO}_{4}\right)_{3}$ переходит в $\mathrm{Fe}_{2}\left(\mathrm{HPO}_{4}\right)_{3}$, затем последний в $\mathrm{FePO}_{4}$. Железо и алюминий «отнимают» на себя часть фосфатов, что приводит к расширению диапазона осаждения для кальция (правый график). Если в растворе находится только $\mathrm{Ca}_{3}\left(\mathrm{PO}_{4}\right)_{2}$ и $\mathrm{FePO}_{4}$ при концентрациях, приведенных в таблице 2, минимальное содержание фосфора $\mathrm{C}_{\mathrm{P}, 0}$ будет равно 0.22 моль/дм ${ }^{3}$. Осаждение солей кальция начинается при концентрации $\mathrm{C}_{\mathrm{P}, 0}$ равной 0.34 моль/дм ${ }^{3}$ и ниже (вертикальная черта на правом графике рисунка 3). Добавление кислоты из вытесняющего раствора в раствор солей приведет к повышению СР,0 до 0.32 моль/дм ${ }^{3}$, что ниже 0.34. Это означает, что отсутствие осаждения в эксперименте нельзя объяснить только лишь образованием более растворимых кислых солей, связанным с повышением концентрации фосфорной кислоты. Вероятным механизмом, который препятствует образованию осадка, может являться эффект изотермического пересыщения. Из эксперимента известно, что при концентрациях вытесняющего раствора ниже 0.1 моль/дм ${ }^{3}$ соли кальция выпадали в осадок, отсюда можно примерно оценить, какой вклад 


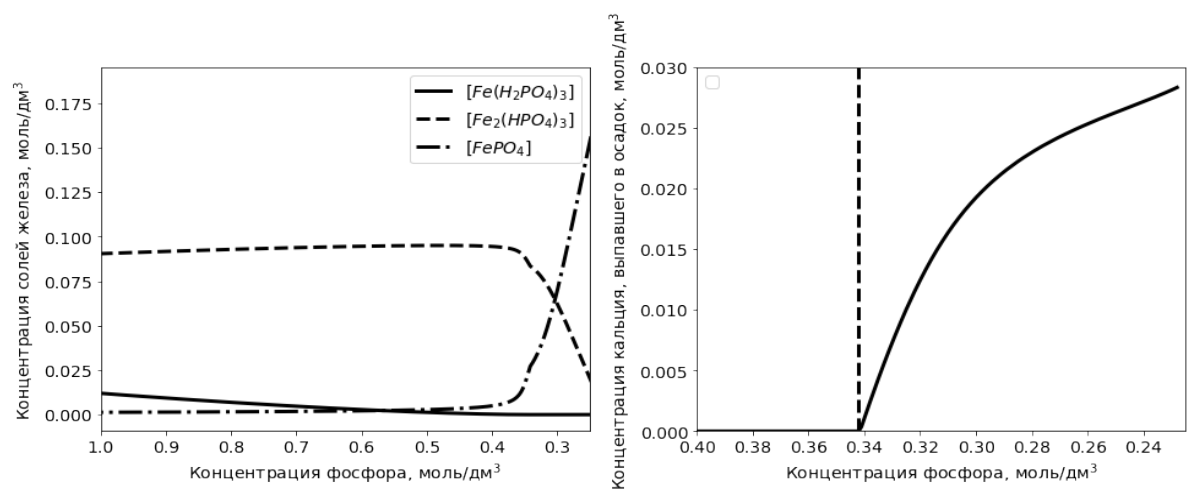

Рис. 3. Результаты моделирования при наличии солей железа.

Fig. 3. Simulation results in the presence of iron salts.

дает эффект изотермического пересыщения. По сравнению с экспериментом, в котором колонка промывалась водой и осадок выпадал, в эксперименте с промывкой слабым раствором кислоты вклад влияния эффекта изотермического пересыщения в стабилизацию процесса составляет более $21 \%$. Так, например, в отсутствие этого эффекта для предотвращения осаждения потребовалось бы использовать промывочной раствор с концентрацией не менее 0.13 моль/дм².

\section{Заключение}

Наличие примесных соединений металлов, в частности, железа и алюминия, оказывает существенное воздействие на молекулярный и ионный состав смеси, перерабатываемой в модифицированном методе удерживания кислоты. Из представленных расчетов видно, что отсут-

\section{Список литературы}

1. Hatch M.J., Dillon J.A. // I\&EC Process Design and Development. 1963. Vol. 2. No 4. pp. 253-264. DOI: https://doi.org/10.1021/i260008a001

2. Ferapontov N.B., Parbuzina L.R., Gorshkov V.I., Strusovskaya N.L. et al. // Reactive and Functional Polymers. 2000. Vol. 45. Issue 2. pp. 145-153. DOI: https://doi.org/10.1016/ S1381-5148(00)00023-7

3. Davankov V., Tsyurupa M., Blinnikova Z., Pavlova L. // Journal of Separation Science. 2009. Vol. 32. Issue 1. pp. 64-73. DOI: https://doi.org/10.1002/jssc.200800449 ствие осадка солей кальция в смеси фосфорной кислоты и солей кальция, железа и алюминия не может быть объяснено только образованием более растворимых кислых солей, что свидетельствует о влиянии эффекта изотермического пересыщения. При вытеснении водой в стандартном методе удерживания кислоты вклада эффекта изотермического пересыщения недостаточно для того, чтобы препятствовать образованию осадка. В случае же промывки слабым раствором фосфорной кислоты совместное влияние образования кислых солей и эффекта изотермического пересыщения становится достаточным для предотвращения осаждения. Дальнейшее исследование требует динамического моделирования внутри колонки с сорбентом с использованием разработанных ранее моделей $[18,19]$, что будет сделано в последующих работах.

4. Хамизов Р.Х. Крачак А.Н., Груздева А.Н. // Сорбиионные и хроматографические nрочессы. 2012. T. 12. № 1. C. 29-39.

5. Власовских Н.С. Хамизов С.Х., Хамизов Р.Х. и др. // Сорбиионные и хромотографические проиессы. 2013. Т. 13. № 5. С. 605-617.

6. Глотова Е.А., Тихонов Н.А., Хамизов P.Х., Крачак А.Н. // Вестник МГУ. Серия 3: Физика. Астрономия. 2013. № 1. С. 64-68.

7. Сидельников Г.Б., Тихонов Н.А., Хамизов Р.Х., Крачак А.Н. // Математическое моделирование. 2013. Т. 25. № 4. С. 3-16.

8. Khamizov R.Kh., Tikhonov N.A., Krachak A.N. et al. // Geochemistry International. 2016. Vol. 54. No 13. Pp. 1221-1235. 
9. Khamizov R.Kh., Myasoedov B.F., Tikhonov N.A., Rudenko B.A. // Doklady Phys. Chemistry. 1997. Vol. 356. No 1-3. pp. 310-314.

10.Tikhonov N.A. // Journal of Mathematical Chemistry. 2019. Vol. 57. pp. 315-326. DOI: https://doi.org/10.1007/s10910-018-0953-y

11.Буцкус Е.Б. в сб. Труды НИУИФ. 1943. Вып.153. С. 110-115.

12.Никольский Б.П., Григоров О.Н., Позин М.Е. и др. Справочник химика. 2-е издание. Т. 3. Химическое равновесие и кинетика растворов, электродные процессы. М. Химия. 1965. $1008 \mathrm{c}$.

13.Лурье Ю.Ю. Справочник по аналитической химии, издание четвертое. М. Химия. $1971.456 \mathrm{c}$.

14.Galal-Gorchev H., Stumm W. // Journal of Inorganic and Nuclear Chemistry. 1963. Vol. 25. Issue 5. pp. 567-574. DOI: https://doi.org/10.1016/0022-1902(63)80243-2
15.Smith S., Takacs I., Murthy S., Daigger G. // Water Environment Research. 2008. Vol. 80. Issue 5. pp. 428-438. DOI: 10.1002/j.15547531.2008.tb00349.x

16. Brosheer J.C., Lenfesty E.A., Anderson J.F. // J. Am. Cem. Soc. 1954. Vol. 76. No 23. pp. 5951-5956. DOI: https://doi.org/10.1021/ja01652a016

17.Калиткин Н.Н., Альшина Е.А. Численные методы. Кн. 1. Численный анализ. М. Академия. 2013. 304 с.

18.Тихонов Н.А., Хамизов Р.Х., Киршин В.В. // Ж. физ. химии. 2000. Т. 74. № 2. С. 309315.

19. Muraviev D., Khamizov R.K., Tikhonov N.A. // Langmuir. 2003. Vol. 19. No 26. pp. 10852-10856. DOI: https://doi.org/10.1021/la030216p

\title{
Influence of impurity components on the precipitation of calcium salts during the purification of phosphoric acid on anionite by the method of acid retardation
}

\author{
(C) 2021 Kaznacheev M.A. ${ }^{1}$, Tikhonov N.A. ${ }^{1}$, Khamizov R.Kh. ${ }^{2}$ \\ ${ }^{1}$ Lomonosov Moscow State University, Moscow \\ ${ }^{2}$ Vernadsky Institute of Geochemistry and Analytical Chemistry of the Russian Academy of Sciences, Moscow
}

The method of acid retardation in a sorbent is effective for the separation of mixtures of acids and salts. It is based on the difference in size of the molecules of acids and hydrated ion pairs of salt components which are formed in highly concentrated solutions. The method involves flowing a solution of a mixture of components with a common anion through a column with a gel anion exchanger in the same anionic form, which allows for the exclusion of the ion exchange. During the first stage of the process, the acid is retained in the sorbent nanopores, while the salt solution flows through the layer. This process continues until the acid retardation ceases and the acid appears at the exit of the column. Then the second stage is carried out: the acid is displaced by washing the layer in the column with water (a classical method) or with a special washing solution (a modified method). As a result, a solution of pure and highly concentrated acid is obtained at the outlet. This work analyses the results of an experiment aimed at the purification of industrial extract phosphoric acid (EPA) with a large amount of impurities by a modified method. In this method the dilute phosphoric acid solution used as a displacing solution prevents the precipitation of calcium salts. To study the effect of the washing solution on the precipitation process, a mathematical model of chemical equilibrium in a system of a limited number of EPA components was formulated. This model allows calculating the molecular and ionic composition of the mixture depending on the concentrations of the input substances, including the determination of the substance amount in the sediment. It was shown that in the case when only calcium salts and phosphoric acid are present in the initial solution the concentration of the washing solution of $0.1 \mathrm{~mol} / \mathrm{dm}^{3}$ for phosphoric acid is sufficient to prevent precipitation. This effect is due to the formation of more soluble acid salts. If the solution contains salts of other metals, in particular aluminium and iron, according to the model's predictions, the mechanism of acid salt formation does not explain the absence of precipitation. This would require a washing solution with a concentration of at least $0.13 \mathrm{~mol} / \mathrm{dm}^{3}$. The simulation's results suggest that other mechanisms are also involved in the prevention of sedimentation. One such mechanism may be the effect of isothermal supersaturation in the ion exchanger layer.

Keywords: the method of acid retardation, separation of salts and acids, chemical equilibrium, mathematical modelling, the effect of isothermal supersaturation. 


\section{References}

1. Hatch M.J., Dillon J.A., I\&EC Process Design and Development, 1963, Vol. 2, No 4, pp. 253-264. DOI: https://doi.org/10.1021/i260008a001

2. Ferapontov N.B., Parbuzina L.R., Gorshkov V.I., Strusovskaya N.L. et al., Reactive and Functional Polymers, 2000, Vol. 45, Issue 2, pp. 145-153, DOI: https://doi.org/10.1016/S13815148(00)00023-7

3. Davankov V., Tsyurupa M., Blinnikova Z., Pavlova L., Journal of Separation Science, 2009, Vol. 32, Issue 1, pp. 64-73. DOI: https://doi.org/10.1002/jssc.200800449

4. Khamizov R.Kh., Krachak A.N., Gruzdeva A.N., Sorbtsionnye $i$ khromatograficheskie protsessy, 2012, Vol. 12, No 1, pp. 29-39.

5. Vlasovskikh N.S., Khamizov S.Kh., Khamizov R.Kh. et al., Sorbtsionnye i khromatograficheskie protsessy, 2013, Vol. 13, No 5, pp. 605-617.

6. Glotova E.A., Tikhonov N.A., Khamizov R.K., Krachak A.N., Moscow Univ. Phys. Bul., 2013, Vol. 68, No 1, pp. 65-70.

7. Sidelnikov G.B., Tikhonov N.A., Khamizov R.K., Krachak A.N., Math. Models Comp. Simulations, 2013, Vol. 5, No 6, pp. 501-510.

8. Khamizov R.Kh., Tikhonov N.A., Krachak A.N. et al., Geochemistry International, 2016, Vol. 54, No 13, pp 1221-1235.

9. Khamizov R.Kh., Myasoedov B.F., Tikhonov N.A., Rudenko B.A., Doklady Phys. Chemistry, 1997, Vol. 356, No 1-3, pp. 310-314.

Казначеев Михаил Александрович - аспирант кафедры математики, физический факультет Московского Государственного Университета имени М.В. Ломоносова, Москва

Тихонов Николай Андреевич - д.ф.-м.н., профессор кафедры математики, физический факультет Московского Государственного Университета имени М.В. Ломоносова, Москва

Хамизов Руслан Хажсетович - д.Х.н., главный научный сотрудник, заместитель директора по науке Института геохимии и аналитической химии имени В.И. Вернадского Российской академии наук, Москва
10. Tikhonov N.A., Journal of Mathematical Chemistry, 2019, Vol. 57, pp. 315-326. DOI: https://doi.org/10.1007/s10910-018-0953-y

11.Butskus E.B., In the collection Proceedings of NIUIF, 1943, Is. 153, pp. 110-115.

12.Nikolsky B.P., Grigorov O.N., Pozin M.E. et al. Chemist's Handbook, Vol. 3, Chemical equilibrium and kinetics of solutions, electrode processes. M., Chemistry, 1965, $1008 \mathrm{p}$.

13. Lurie Yu.Yu. Analytical Chemistry Handbook, M., Chemistry, 1971, 456 p.

14.Galal-Gorchev H., Stumm W., Journal of Inorganic and Nuclear Chemistry, 1963, Vol. 25, Issue 5, pp. 567-574, DOI: https://doi.org/10.1016/0022-1902(63)80243-2

15.Smith S., Takacs I., Murthy S., Daigger G., Water Environment Research, 2008, Vol. 80, Issue 5, pp. 428-438. DOI: $10.1002 / \mathrm{j} .1554-$ 7531.2008.tb00349.x

16. Brosheer J.C., Lenfesty E.A., Anderson J.F., J. Am. Cem. Soc., 1954, Vol. 76, No 23, pp. 5951-5956. DOI: https://doi.org/10.1021/ja01652a016

17.Kalitkin N.N., Alshina E.A. Numerical Methods, Book 1, Numerical Analysis. M., Academy, 2013, 304 p.

18. Tikhonov N.A., Kirshin V.V., Khamizov R.Kh., Russian J. Phys. Chemistry A, 2000, Vol. 74, No 2, pp. 246-252.

19.Muraviev D., Khamizov R.K., Tikhonov N.A., Langmuir, 2003, Vol. 19, No 26, pp. 1085210856. DOI: https://doi.org/10.1021/la030216p

Kaznacheev Mikhail A. - postgraduate student, department of mathematics, Faculty of Physics, Moscow State University, Moscow, kaznacheev.michael@mail.ru

Tikhonov Nikolay A. - prof., grand Ph.D. (Phys.-Math.), department of mathematics, Faculty of Physics, Moscow State University, Moscow, niktandr@yandex.ru

Khamizov Ruslan Kh. - grand Ph.D. (Chemistry), chief researcher, Deputy Director for Science of the Vernadsky Institute of Geochemistry and Analytical Chemistry of Russian Academy of Sciences, Moscow, khamiz@mail.ru 